\title{
Sentiment Classification based on Domain Prediction
}

\author{
Muhammet Yasin $\mathrm{Pak}^{1}$, Serkan Gunal ${ }^{1}$ \\ ${ }^{I}$ Department of Computer Engineering, Anadolu University, \\ Eskisehir, Turkiye \\ mypak@anadolu.edu.tr
}

\begin{abstract}
Sentiment classification has received increasing attention in recent years. Supervised learning methods for sentiment classification require considerable amount of labeled data for training purposes. As the number of domains increases, the task of collecting data becomes impractical. Therefore, domain adaptation techniques are employed. However, most of the studies dealing with the domain adaptation problem demand a few amount of labeled data or lots of unlabeled data belonging to the target domain, which may not be always possible. In this work, a novel method for sentiment classification, which does not require labeled and/or unlabeled data from the target domain, is proposed. The propose method mainly consists of two stages. At first, the target domain is predicted even if it is not among the source domains in hand. Then, sentiment is classified as either positive or negative using the sentiment classifier specifically trained for the predicted domain. Extensive experimental analysis on two different datasets with distinct languages and domains verifies that the proposed method is superior to the domain independent sentiment classification approach at each case considered.
\end{abstract}

Index Terms-Sentiment analysis; sentiment classification; text classification; domain adaptation.

\section{INTRODUCTION}

Nowadays, most people share their opinions through the internet on which the products and services they buy or use. While they give credit to some of those products and services, they may also make criticizing comments as well. Sentiment analysis, also called as opinion mining, fundamentally aims to extract positive or negative sentiment present on those shared opinions via text processing. Sentiment analysis determines the positive, negative or neutral feeling of opinion holders about the entity and the aspects of entity within the given text.

While a number of studies tackle the sentiment classification problem at the aspect level, most of the works focus on the document level classification. In case of document level classification, it is assumed that a document expresses an opinion on a single entity and detect general sentiment rather than the sentiment of each aspect [1].

While there are some unsupervised methods proposed for sentiment classification [2], most of the existing approaches are based on supervised learning [3]. Supervised learning methods need a large amount of labeled data to train the classifier. Besides, sentiment classification is a domain

Manuscript received 21 November, 2015; accepted 26 March, 2016. dependent problem [4], [5]. For instance, a sentiment classifier used for the reviews in movie domain does not offer the same performance for the reviews in electronics domain. For this reason, a successful classification would require to model the classifier by collecting labeled data for each new domain. Considering that the data collection is a costly process, it is not always possible to collect data for each domain. Instead, existing data rather than collecting new one may be used to minimize the need for labeled data to train the classifier on a new domain. This approach is called as domain adaptation [4]. Several methodologies are proposed for the domain adaptation in sentiment classification. In domain adaptation, the new domain to be classified is expressed as the target domain while the existing domains with the labeled data are expressed as the source domains. While some studies dealing with the domain adaptation problem require just a few labeled data belonging to the target domain, some others need lots of unlabeled data on the target domain. One or more domains can be used as the source domains [6].

In this work, a novel method for sentiment classification, which does not require neither labeled nor unlabeled data on the target domain, is proposed. In this two-stage method, the target domain is first predicted. Then, sentiment classification is carried out considering the predicted target domain. While evaluating the effectiveness of the proposed method, two distinct datasets with different languages, namely Turkish and English, are employed. Experimental analysis verifies that the proposed method beats the domain independent sentiment classification approaches.

The rest of the paper is organized as follows: In Section II, the related works in the literature are briefly reviewed. In Section III, the proposed method is introduced. Section IV explains the datasets employed during the experiments. In Section V, the experimental work and the related results are presented. Finally, some concluding remarks are provided in Section VI.

\section{RELATED WORK}

The domain adaptation approaches based on multiple sources are simply categorized into two groups, namely feature representation and the combination of the prelearned classifiers. Feature representation approaches change the representation of features to make the target and source domains similar by decreasing the weight of different features while increasing the weight of similar ones. The 
approaches based on the combination of the prelearned classifiers offer solutions by combining the classifiers that are trained for each domain. Here, the key point is how to select the classifiers and to determine appropriate weights for them.

In a study on the domain adaptation for sentiment classification, four approaches are investigated for a new target domain in the absence of large amount of labeled data [5]. These approaches are using the classifier trained by combining the labeled data of all source domains, the same classifier by limiting with the features observed in the target domain, combination of the classifiers from each source domains, the expectation maximization algorithm with small amount of labeled data and large amount of unlabeled data on the target domain. In another work, the structural correspondence learning algorithm that is used for domain adaptation is extended to sentiment classification [4]. Pivot features between the source and target domains are selected and it is also showed how to correct structural correspondence misalignments by using a small amount of labeled data on the target domain. In [7], a 2-stage method is proposed such that generalizable features between the target and source domains are extracted in the first stage while the second stage aims to find specific features to target domain using semi-supervised learning methods. A novel method, called as joint sentiment topic model based on latent Dirichlet allocation [8], is proposed in [9]. With this model, sentiment and topic can be detected simultaneously so that the system becomes portable on inter domains for sentiment classification. Spectral feature alignment algorithm is proposed in [10] to bridge the gap between domains. This algorithm uncovers the association between domain specific words by matching with the help of domain independent words. In [11], sentiment sensitive thesaurus is created using labeled and unlabeled data from multiple source domains and unlabeled data from the target domain. In this way, the association between the words that express similar sentiments in different domains are revealed and this knowledge is used to expand the feature vector. In another study [12] with multiple source domains, lexicon similarity is computed between the target and source domains to find the most similar source domain. Also, ensemble classifier with the source domains is modeled by considering the weights computed using lexicon similarity.

\section{The Proposed Method}

As mentioned in the related work, most of the methods developed for domain adaptation problem in sentiment classification require labeled or unlabeled data on the target domain. However, it is not possible to collect any data when the target domain is unknown. In this case, the problem of how to use existing source domains arises.

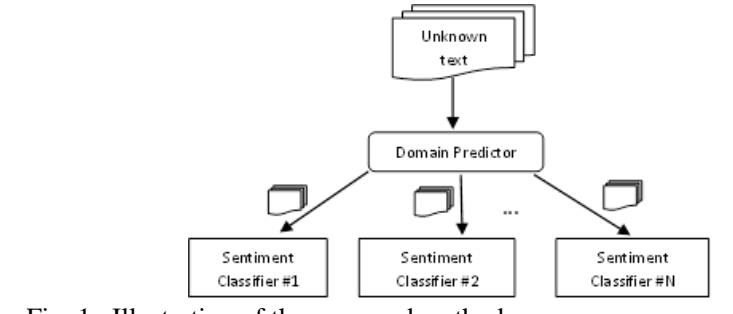

Fig. 1. Illustration of the proposed method.
In this work, a novel method, which does not require any data on the target domain, is proposed for sentiment classification problem. The proposed method fundamentally consists of two stages. In the first stage, the target domain is predicted. Then, in the second stage, sentiment classification is carried out considering the predicted target domain. More specifically, the first stage aims to determine the domain of an unknown data. If the unknown data belongs to one of the existing source domains, it will be assigned to the belonging domain. If the unknown data does not belong to any of the source domains, it will be assigned to the most similar source domain. In the second stage, sentiment classification of the unknown data is realized using the sentiment classifier specifically trained for the source domain that is predicted in the first stage. Domain prediction stage can be considered as an $\mathrm{N}$-class text classification problem for $N$ source domains. In the sentiment classification stage, unknown text is classified into one of two classes, namely positive or negative. Sentiment classifiers are constructed for each source domain by just using the labeled data within that domain. Thus, an unknown text is first classified into one of $N$ source domains. Sentiment classifier on the specified source domain is then used to determine the sentiment of the unknown text. The proposed method is illustrated in Fig. 1.

\section{DATASETS}

The proposed method is evaluated using two different datasets containing the texts from two distinct languages, namely Turkish and English. Turkish is a good example of agglutinative languages whereas English is a nonagglutinative one. Due to the nature of these languages, sentiment statements are completely different. In this way, the effectiveness of the proposed method can be observed on both languages. English dataset contains product reviews from five domains including book, DVD, electronics, health and kitchen. There are 1.000 positive and 1.000 negative reviews for each domain. For Turkish language, a brand new dataset was constituted. For this purpose, product reviews from particular e-commerce websites were collected and labeled with the help of user ratings. The domains in Turkish dataset are computer, cosmetics, auto accessories, phone and movie. Each domain contains 350 positive and 350 negative reviews.

\section{EXPERIMENTAL WORK}

During the experiments, stopword removal, lowercase conversion and stemming were applied as the fundamental preprocessing steps. Stemming for Turkish texts is handled by the natural language processing library for Turkish, named as Zemberek [13]. Negative words are treated as "neg_word" to indicate the oppositeness. The bag-of-words model [14]-[16] was used to represent the text documents, where the exact order of the words, or terms, in the documents was ignored but the occurrences of the terms were considered. Hence, each unique term was handled as a feature. Emoticons expressing positive or negative sentiment were also used as the features. Terms were individually assigned certain weights that represent the importance of the terms. For this purpose, term frequency-inverse document 
frequency (TF-IDF) term weighting schemes was preferred. TF-IDF scales down the number of occurrences of a term in a given text by considering the number of documents in the collection containing the regarding term [15], [17]. The distinctive features (terms) are determined by using the information gain [15], which is one of the successful filterbased feature selection methods. After an empirical verification, the first $20 \%$ of the entire feature set was used to constitute the feature vectors. As a reference, top-10 distinctive terms for each class in two datasets are respectively listed in Table I.

TABLE I. TOP-10 DISTINCTIVE TERMS OF EACH CLASS IN

\begin{tabular}{|c|c|}
\hline Class & (A) Terms \\
\hline Computer & $\begin{array}{c}\text { tavsiye, değil, eder, fakat, teşekkür, gayet, ancak, ama, } \\
\text { ulaş, bellek }\end{array}$ \\
\hline Cosmetics & $\begin{array}{l}\text { değil, eder, ama, tavsiye, yorum, firça, taş, de, fiyat, } \\
\text { fakat }\end{array}$ \\
\hline $\begin{array}{l}\text { Auto } \\
\text { Access. }\end{array}$ & $\begin{array}{l}\text { değil, eder, ama, mükemmel, fena, gayet, tavsiye, } \\
\text { herkes, neg_et, güzel }\end{array}$ \\
\hline Phone & $\begin{array}{c}\text { ses, teşekkür, karşıla, ihtiyaç, değil, ödem, koşul, harika, } \\
\text { fakat, ama }\end{array}$ \\
\hline Movie & $\begin{array}{c}\text { kötü, süper, harika, berbat, mükemmel, yazık, para, } \\
\text { güzel, izle, smile }\end{array}$ \\
\hline Class & (B) Terms \\
\hline Book & $\begin{array}{c}\text { author, war, excellent, don't, boring, not, also, nothing, } \\
\text { life, waste }\end{array}$ \\
\hline DVD & $\begin{array}{c}\text { season, bad, boring, was, nothing, worst, not, seagal, } \\
\text { waste, commander }\end{array}$ \\
\hline Electronics & $\begin{array}{c}\text { great, not, support, customer, highly, price, easy, return, } \\
\text { router, told }\end{array}$ \\
\hline Health & $\begin{array}{c}\text { not, love, great, oil, return, best, flaxseed, waste, easy, } \\
\text { money }\end{array}$ \\
\hline Kitchen & $\begin{array}{c}\text { easy, not, perfect, love, return, great, back, waste, after, } \\
\text { excellent }\end{array}$ \\
\hline
\end{tabular}

Naïve Bayesian (NB) and Support Vector Machine (SVM) classification algorithms [18] were employed for the domain prediction and sentiment classification purposes. Eclipse environment was used during the experiments. Specifically, LIBSVM library was utilized to perform SVM classification whereas a custom software was developed for NB classifier.

TABLE II. NB/SVM CLASSIFICATION RESULTS (\%) WHEN THE TARGET DOMAIN IS AMONG THE SOURCE DOMAINS.

\begin{tabular}{|c|c|c|}
\hline Dataset & Domain Independent & Proposed Method \\
\hline Turkish & $74.06 / 75.42$ & $75.43 / 76.69$ \\
\hline English & $73.60 / 78.22$ & $77.15 / 80.77$ \\
\hline
\end{tabular}

The experiments were carried out by considering two possible cases. In the first case, the target domain was supposed to be among the source domains. According to this assumption, a domain independent sentiment classifier was trained with the labeled data of all source domains including the target domain itself. Sentiment of an unknown text was then determined by this classifier. This approach is similar to the one introduced in [5]. Later, the proposed method was utilized so that the target domain of the unknown text was first predicted, and the sentiment of the text was then determined using the domain specific sentiment classifiers. Sentiment classification results of both approaches for Turkish and English datasets are comparatively presented in Table II, where the highest values are indicated in bold. It is clear from this table that the proposed method surpasses the domain independent sentiment classification for all the languages and classifiers. The highest accuracies in Turkish and English datasets were attained by SVM classifier as $76.69 \%$ and $80.77 \%$, respectively.

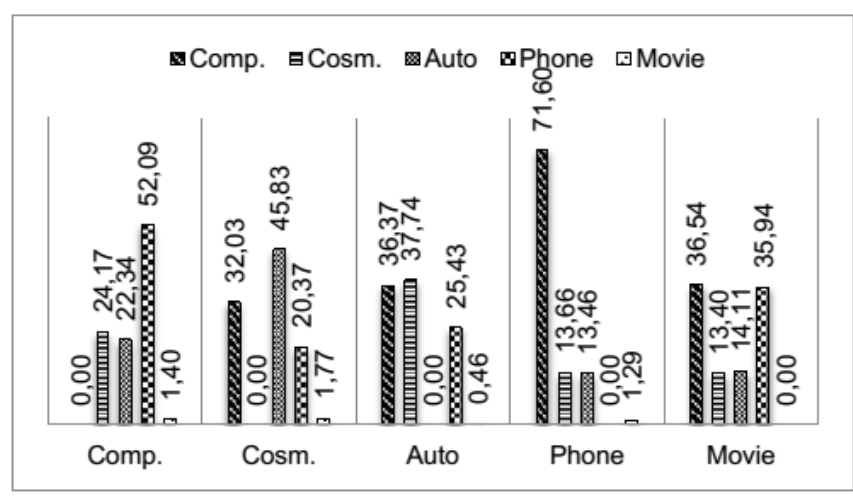

(a)

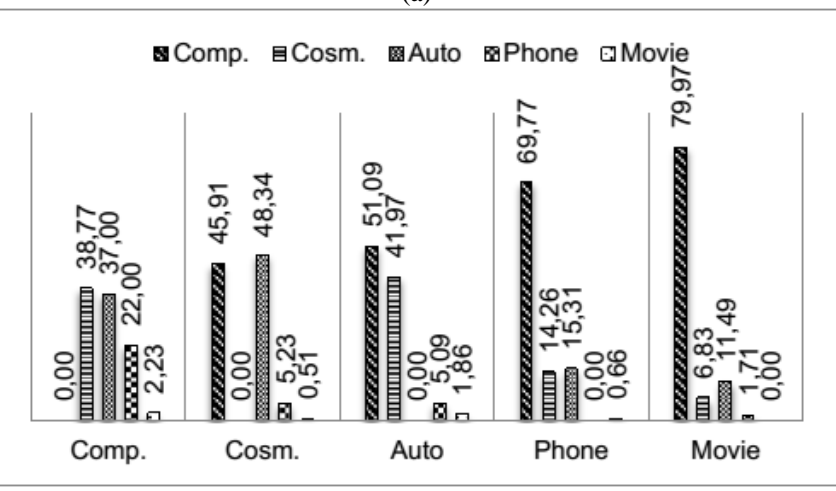

(b)

Fig. 2. Domain prediction results (\%) for Turkish dataset computed by: (a) NB; (b) SVM classifier.

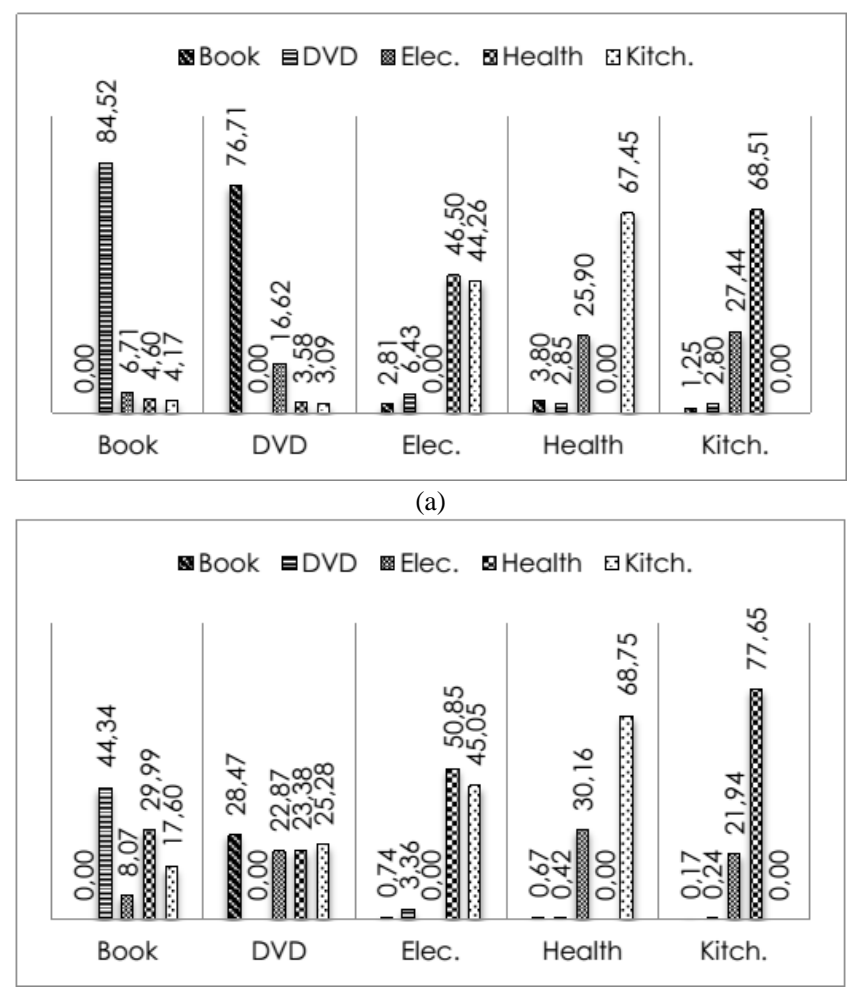

(b)

Fig. 3. Domain prediction results (\%) for English dataset computed by: (a) NB; (b) SVM classifier.

The second case of the experiments assumed that the target domain does not exist within the source domains. Just like the first case, a domain independent sentiment classifier 
was first trained with the labeled data of all source domains. This time, however, no labeled data from the target domain was used for the training. For example, in Turkish dataset, if the target domain is Computer, the source domains including Cosmetics, Auto Accessories, Phone and Movie are just used for the training. Next, the proposed method was utilized as in the first case so that the target domain was first predicted. For instance, in English dataset, if the target domain is Book, the domain predictor would assign a text from Book domain to the most similar domain among DVD, Electronics, Health and Kitchen domains. Domain prediction results for Turkish and English datasets are shown in Fig. 2 and Fig. 3, respectively. One can observe from those figures that the highest similarities are present between Movie and Computer domains (79.97 \%) in Turkish dataset with SVM classifier, and between Book and DVD domains $(84.52 \%)$ in English dataset with NB classifier.

Sentiment of the text was then determined using the sentiment classifier for the predicted domain. Sentiment classification results related to this part of the experiments are listed in Table III, where the highest values are indicated in bold. The proposed method beats the former approach most of the time. The only exceptions were Auto Accessories domain in Turkish dataset and DVD domain in English dataset when SVM classifier was used.

TABLE III. NB/SVM CLASSIFICATION RESULTS (\%) WHEN THE TARGET DOMAIN IS NOT AMONG THE SOURCE DOMAINS IN (A) TURKISH (B) ENGLISH DATASET.

\begin{tabular}{|c|c|c|}
\hline (A) Target Domain & Domain Independent & Proposed Method \\
\hline Computer & $73.63 / 74.74$ & $\mathbf{7 6 . 3 4} / 75.60$ \\
\hline Cosmetics & $75.29 / 76.37$ & $77.23 / 78.11$ \\
\hline Auto Accessories & $74.11 / 77.89$ & $77.09 / 77.37$ \\
\hline Phone & $74.26 / 74.91$ & $\mathbf{7 6 . 0 6} / 75.31$ \\
\hline Movie & $66.69 / 64.57$ & $\mathbf{6 7 . 8 6} / 64.91$ \\
\hline (B) Target Domain & Domain Independent & Proposed Method \\
\hline Book & $72.65 / 71.59$ & $\mathbf{7 5 . 8 0} / 73.30$ \\
\hline DVD & $74.82 / 75.46$ & $\mathbf{7 6 . 0 0 / 7 4 . 7 3}$ \\
\hline Electronics & $75.12 / 75.46$ & $77.43 / 79.05$ \\
\hline Health & $76.13 / 77.50$ & $76.97 / 79.10$ \\
\hline Kitchen & $77.82 / 78.75$ & $80.27 / 82.19$ \\
\hline
\end{tabular}

\section{CONCLUSIONS}

A novel method for sentiment classification is proposed to resolve specifically the domain adaptation problem. While most of the related studies in the literature demand labeled and/or unlabeled data for the target domain, the proposed method does not have such a requirement. In this new method, target domain is first predicted even if it is not among the source domains available. Then, sentiment classification is carried out by considering the predicted domain. Experimental analysis verifies that the proposed method offers superior performance with respect to the domain independent approach even for different languages and classification algorithms studied on. The effectiveness of the proposed method is valid whether the target domain is among the source domains or not. However, the performance of the classifiers against each other may vary depending on the domain and language. For instance, NB offers higher accuracy than SVM when the target domain is Computer in Turkish dataset. On the other hand, SVM is better than NB if the target domain is Electronics in English dataset.

The proposed approach can be easily extended to other domains and languages as well. Analysis of different classification algorithms and feature selection approaches for the proposed framework remain as interesting future works.

\section{REFERENCES}

[1] B. Liu, Sentiment Analysis and Opinion Mining. Morgan and Claypool, 2012.

[2] P. D. Turney, "Thumbs up or thumbs down?: semantic orientation applied to unsupervised classification of reviews", in Proc. 40th Annual Meeting on Association for Computational Linguistics, 2002, pp. 417-424. [Online]. Available: http://dx.doi.org/10.3115/1073083. 1073153

[3] B. Pang, L. Lee, S. Vaithyanathan, "Thumbs up? sentiment classification using machine learning techniques", in Proc. of ACL-O2 Conf. on Empirical Methods in Natural Language Proc., 2002, pp. 79-86. [Online]. Available: http://dx.doi.org/10.3115/1118693. 1118704

[4] J. Blitzer, M. Dredze, F. Pereira, "Biographies, bollywood, boomboxes and blenders: domain adaptation for sentiment classification", in Proc. 45th Annual Meeting of the Association for Computational Linguistics, 2007, pp. 440-447.

[5] A. Aue, M. Gamon, "Customizing sentiment classifiers to new domains: a case study", in Proc. Int. Conf. on Recent Advances in Natural Language Processing, 2005.

[6] S. Sun, H. Shi, Y. Wu, "A survey of multi-source domain adaptation", Information Fusion, vol. 24, pp. 84-92, 2015. [Online]. Available: http://dx.doi.org/10.1016/j.inffus.2014.12.003

[7] J. Jiang, C. Zhai, "A two-stage approach to domain adaptation for statistical classifiers", in Proc. 16th ACM Conf. Information and Knowledge Management, 2007, pp. 401-410. [Online]. Available: http://dx.doi.org/ 10.1145/1321440.1321498

[8] D. M. Blei, A. Y. Ng, M. I. Jordan, "Latent Dirichlet allocation", Journal of Machine Learning Research, vol. 3, pp. 993-1022, 2003.

[9] C. Lin, Y. He, R. Everson, S. Ruger, "Weakly supervised joint sentiment-topic detection from text", IEEE Trans. on Knowledge and Data Engineering, vol. 24, no. 6, pp. 1134-1145, 2012. [Online]. Available: http://dx.doi.org/10.1109/TKDE.2011.48

[10] S. J. Pan, X. Ni, J. T. Sun, Q. Yang, Z. Chen, "Cross-domain sentiment classification via spectral feature alignment", in Proc. 19th Int. Conf. on World Wide Web, 2010, pp. 751-760. [Online]. Available: http://dx.doi.org/10.1145/1772690.1772767

[11] D. Bollegala, D. Weir, J. Carroll, "Using multiple sources to construct a sentiment sensitive thesaurus for cross-domain sentiment classification", in Proc. 49th Annual Meeting of the Association for Computational Linguistics, 2011, pp. 132-141.

[12] M. Whitehead, L. Yaeger, "Building a general purpose cross-domain sentiment mining model", in Proc. WRI World Congress on Computer Science and Information Engineering, vol. 4, 2009, pp. 472-476. [Online]. Available: http://dx.doi.org/ 10.1109/CSIE.2009.754

[13] A. A. Akin, M. D. Akin, "Zemberek, an open source NLP framework for Turkic languages", 2007.

[14] T. Joachims, "A probabilistic analysis of the Rocchio algorithm with TFIDF for text categorization", in Proc 14th Int. Conf. on Machine Learning, 1997, pp. 143-151.

[15] S. Gunal, "Hybrid feature selection for text classification", Turkish Journal of Electrical Engineering \& Computer Sciences, vol. 20, no. 2, pp. 1296-1311, 2012. [Online]. Available: http://dx.doi.org/ 10.3906/elk-1101-1064

[16] A. K. Uysal, S. Gunal, "A novel probabilistic feature selection method for text classification”, Knowledge-Based Systems, vol. 36, pp. 226-235, 2012. [Online]. Available: http://dx.doi.org/10.1016/ j.knosys.2012.06.005

[17] A. K. Uysal, S. Gunal, S. Ergin, E. Sora Gunal, "The impact of feature extraction and selection on SMS spam filtering", Elektronika ir Elektrotechnika, vol. 19, no. 5, pp. 67-72, 2013. [Online]. Available: http://dx.doi.org/10.5755/j01.eee.19.5.1829

[18] S. Theodoridis, K. Koutroumbas, Pattern Recognition, Academic Press, 2008. 